See discussions, stats, and author profiles for this publication at: https://www.researchgate.net/publication/269328750

\title{
Sclera Recognition - A Survey
}

Conference Paper · November 2013

DOI: 10.1109/ACPR.2013.168

CITATIONS

31

4 authors, including:

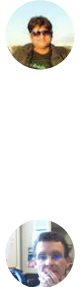

Abhijit Das

University of Southern California

42 PUBLICATIONS 301 CITATIONS

SEE PROFILE

Miguel A. Ferrer

Universidad de Las Palmas de Gran Canaria

281 PUBLICATIONS 2,978 CITATIONS

SEE PROFILE

Some of the authors of this publication are also working on these related projects:

Project Proyecto Fin de Carrera View project

Project $\quad$ PhD project View project
READS

755

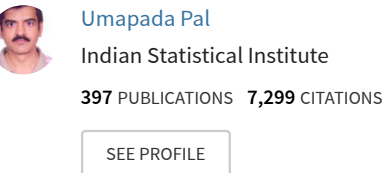




\title{
Sclera Recognition - A Survey
}

\author{
Abhijit Das*, Umapada Pal ${ }^{\dagger}$, Michael Blumenstein* and Miguel Angel Ferrer Ballester ${ }^{\ddagger}$ \\ *Institute for Integrated and Intelligent Systems, Griffith University, Queensland, Australia \\ Email: abhijit.das@griffithuni.edu.au, m.blumenstein@griffith.edu.au \\ †Computer Vision and Pattern Recognition Unit, Indian Statistical Institute, Kolkata, India, Email: umapada@isical.ac.in \\ ${ }_{\ddagger}^{\ddagger}$ IDeTIC, University of Las Palmas de Gran Canaria, Las Palmas, Spain, Email: mferrer@dsc.ulpgc.es
}

\begin{abstract}
This paper presents a survey on sclera-based biometric recognition. Among the various biometric methods, sclera is one of the novel and promising biometric techniques. The sclera, a white region of connective tissue and blood vessels, surrounds the iris. A survey of the techniques available in the area of sclera biometrics will be of great assistance to researchers, and hence a comprehensive effort is made in this article to discuss the advancements reported in this regard during the past few decades. As a limited number of publications are found in the literature, an attempt is made in this paper to increase awareness of this area so that the topic gains popularity and interest among researchers. In this survey, a brief introduction is given initially about the sclera biometric, which is subsequently followed by background concepts, various pre-processing techniques, feature extraction and finally classification techniques associated with the sclera biometric. Benchmarking databases are very important for any pattern recognition related research, so the databases related with this work is also discussed. Finally, our observations, future scope and existing difficulties, which are unsolved in sclera biometrics, are discussed. We hope that this survey will serve to focus more researcher attention towards the emerging sclera biometric.
\end{abstract}

Keywords: sclera biometric, pattern, Recognition, survey.

\section{INTRODUCTION}

Biometrics refers to automatic authentication of individuals based on their physiological and behavioural characteristics [1]. Over the last few decades, intensive research work has been performed in the field of biometrics to identify individuals as accurately as possible based on biological or physiological traits. But it was found that no biometric technique is perfect or can be applied universally. In order to increase population coverage, extend the range of environmental conditions, improve resilience to spoofing, and achieve higher recognition accuracy, further research on biometric traits is required [2].

Among the various biometric techniques, sclera recognition is considered a good trait to complement traditional traits, because sclera areas are highly-protected portions of the eye, which are very difficult to spoof. Identification of a person by the vessel pattern of the sclera is possible because firstly, these patterns possess a high degree of randomness, which is never the same for any two individuals, even for identical twins [3] [4], and this makes it ideal for personal identification. Secondly, the patterns remain stable throughout a person's lifetime [5], these patterns even differ for the right and the left eye of the same individual. It is interesting to note that humans are the only mammals with extensive exposed sclera, which is amenable to imaging of the encompassing conjunctival vasculature [6] and this is another advantage to use sclera for human biometrics. Additionally this trait can be easily combined with iris biometrics. As among biometrics systems, iris recognition is tested to be one of the most reliable approaches for automatic personal recognition. However, the iris patterns could reveal rich and complex patterns only under near-infrared (NIR). If we acquire iris images in visible light, the accuracy of iris recognition will drop dramatically. So the combination of the sclera feature gives a new art to iris biometrics [25].

The various challenges in sclera recognition include accurate segmentation of the sclera area, sclera vessel enhancement and the extraction of discriminative features of the sclera vessel pattern for authentication and identification purposes. The task becomes more difficult as frequently a complete sclera image is not obtained but is occluded by portions of the eyelid and eyelashes. Moreover, different lighting conditions can change the appearance of the texture patterns by accentuating and attenuating various grey tones. Also the authentication system should work in real-time so that extraction, representation and comparison of texture images should not consume large computational resources. After that, a classification system uses the mathematical model of the sclera texture to compare with other sclera images to identify specific individuals or recognise an individual.

The organization of the paper is as follows: - In Section II anatomy of human sclera, Section III background of biometric systems and performance, Section IV provides a detailed literature survey of different techniques of the sclera biometric. Datasets Available and performance on them are explained in section V. Medical and other conditions that can affect the sclera biometric result are highlighted in Section VI. Finally, our observations, future scope and existing difficulties, which are unsolved in sclera biometrics, are discussed in Section VII.

\section{SCLERA ANATOMY}

The sclera, a white region of connective tissue and blood vessels, surrounds the iris. Iris is a colour ring of tissue around the pupil. A clear covering called the cornea covers the iris and the pupil. The pupil region generally appears darker than the iris. However, the pupil may have specular highlights and cataracts can lighten the pupil. The sclera typically has a rich pattern due to the connective tissues and blood vessels in different orientations and layers.

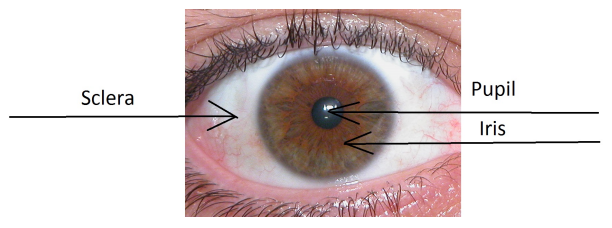

Fig. 1. Colour image of an eye consiting of pupil, iris and sclera area. 


\section{BACK GROUND AND PERFORMANCE OF BIOMETRIC}

Biometrics is used in two different types of application scenario. Firstly, in a verification scenario, a person claims a particular identity and the biometric system is used to verify or reject the claim. Verification is performed by matching a biometric sample acquired at the time of the claim against the sample previously enrolled for the claimed identity. In Identification mode the system performs a one-to-many comparison against a biometric database in attempt to establish the identity of an unknown individual. If the two samples match well enough, the identity claim is verified, and if the two samples do not match well enough, the claim is rejected. Another applications scenario of biometric is recognition. Recognition mode the system performs a one-to-one comparison against a biometric database in attempt to establish the recognition of an unknown individual.

The different measures of a biometric system are mainly FAR (False Acceptance Rate), FRR (False Rejection Rate) and EER (Equal Error Rate). A false acceptance occurs when the system accepts an identity claim, but the claim is not true. A false reject occurs when the system rejects an identity claim, but the claim is true. Biometric performance in a verification scenario is often summarized in a receiver operating characteristic (ROC) curve. The EER is a single number often quoted from the ROC curve. The EER is where the false acceptance rate equals the false rejection rate. The terms verification and authentication are often used interchangeably in this context. Performance in an recognition scenario is often summarized in a cumulative match characteristic (CMC) curve.

\section{DIFFERENT STEPS IN SCLERA BIOMETRIC}

Using vascular patterns for personal identification has been studied in the context of fingers [7], palm [8], and retina [9]. The first recognized work on sclera biometrics using blood vessels of sclera is recorded in [10]. All the contributions of the different works found in sclera literature can be clustered in to four basic categories. They are sclera segmentation, sclera vessel enhancement and image registration, feature extraction and classification. The various techniques of the above mention steps are explained in the following sections. A typical sclera biometric system is explained in Fig. 2.

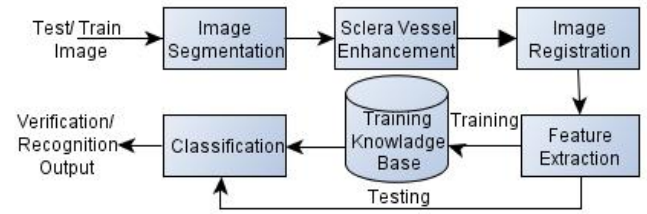

Fig. 2. A typical sclera biometric system with different steps involve.

\section{A. Sclera segmentation}

Segmentation is the first step for most biometric related research. Here the main aim is to identify the region of interest as appropriate as possible. Similarly in sclera biometric a perfect segmentation is important otherwise, an incorrect segmentation can reduce the pattern available, but also it can introduce other patterns such as eyelashes and eyelids. So in the literature of sclera biometric the researchers have given a great importance to this phase. In the initial few works of sclera biometric literature like in [10] and [14] manual segmentation was used.

Possibly [28] was the first work on automated sclera segmentation. Here, the sclera was segmented by a timeadaptive active contour-based method. The iris was localized in the detected eye strip in the binary image through template matching via an adaptive half-circle template. Next, two points on the circular circumference of the iris at angles 80 degree from the vertical line were calculated. Then with a 45 degree angle projection to horizontal in up and down direction was used to find the first points, two points with a large difference in brightness compared to the previous pixel in the path were used to get the two corners of the eye. A TASOM (Time Adaptive Self-Organizing Map)-based active contour method detailed in [29], [30] was used to get the inner boundary of the sclera.

In [40], the authors have designed enhancement and registration methods to process and match conjunctival vasculature obtained under non-ideal conditions. For each eye, 2 images were collected per gaze at each distance. K-means clustering was used here for sclera segmentation. The sclera pixel cluster was determined as that cluster which has the largest Euclidean distance from the origin of the coordinate system to its centroid. Here to detect specular reflections the power law transformation was applied to pixel intensities in the colour image.

In [39], a colour image sclera segmentation process was proposed, which includes image down sampling, conversion to the HSV colour space, estimation of the sclera region, iris and eyelid detection, eyelid and iris boundary refinement, mask creation, and mask up-sampling. In [15], a robust multi-angled sclera recognition technique was proposed. For segmentation a three by three Sobel filter was applied to highlight the wanted glare area in the eye image. The pupil and iris regions are simply modelled as circular boundaries and typical circular iris segmentation methods were used in this research as in [16]-[24]. As the intensity of sclera area is different from the background, for which Otsus method was applied to detect the region of interest. In order to get a finer segmentation boundary, a refinement using the Fourier active contour method [17] was performed. A new robust method of sclera segmentation for colour images was proposed in [2]. For colour images estimation of the centres of pupil and iris was done as in [15] the HSV model was used for segmentation the eye images automatically. A method employed to segment the sclera region along the eyelid contour was inspired by the work performed in the processing of LandSat imagery (Land + Satellite) [32].

A sclera segmentation technique for multi-spectral occluded sclera was proposed in [31]. A set of indices are used to segment the vegetation regions in aerial multispectral images. Similarly, the index that used for coarse sclera segmentation was based on the fact that the skin has lesser water content than the sclera, and hence exhibits a higher reflectance in NIR. Since water absorbs NIR light, the corresponding regions based on this index appear dark in the image. Then an index called the normalized sclera index image was computed and the sclera area was achieved by thresolding. The pupil segmentation method was described in [33] and subsequently the iris boundary was calculated. In the third step using the iris bounder and the sclera boundary was segmented. 


\section{B. Sclera vessel enhancement and Image registration}

The vessels in the sclera are not prominent, so in order to make them clearly visible, image enhancement is required. Various techniques for sclera vessel enhancement are found in literature. The first recognized work on sclera vessels enhancement is recorded in [10]. First of all a contrast-limited adaptive histogram equalization scheme was applied to the region of interest in order to enhance the image. Then the green layer of the RGB image was used. This leads to a better contrast between the blood vessels and the background as explained in [11]. A selective line enhancement technique, as explained in [12], was used to enhance the blood vessels of the green channel. This technique uses the second derivative of the Hessian matrix to enhance the vein pattern. As derivatives are sensitive to noise, the image is first convolved with a Gaussian function with a standard deviation. Here, for every pixel in the image, two eigenvalues of the Hessian matrix are computed. As the veins are of different thickness over the image, so a multi-scale iterative Gaussian enhancement filter with varying standard deviations was used. To obtain a binary representation of the region, the growing algorithm of [13] was used.

In [14], [35], [37],[40] adaptive histogram equalization was applied to the green layer of the colour image in order to get enhanced sclera vain pattern. In [40] for image registration a local affine and a global smooth transformation was applied. A image mapping method was used in [37] to make the images invariant to rotation. In [25] and [39] sclera vain pattern enhancement by Gabor filter. And after morphological operations, image can get enhanced sclera vessel patterns from the original image.

In [39] a poler coordinate system was used to map the image with respect to pupil centre. As the vascular patterns could have multiple orientations, in [2] and [15] a bank of directional Gabor filters was used for vascular pattern enhancement. A polar coordinate-based mapping with iris centre was performed. Using the iris centre as a reference, the line descriptor was translation invariant mapping was performed with the segmented enhanced vessel image. The line descriptor can extract patterns in different orientations, which makes it possible to achieve orientation-invariant matching. In [31] selective enhancement filter for lines, and implicitly for blood vessels, as described in [34] and used in [33] was applied to the green component.

\section{Feature extraction}

The feature extraction of sclera recognition system involves in building a reliable mathematical model of the abstract sclera pattern to reliably identify persons for authentication and identification purposes. In few paper of sclera literature vain enhanced images were directly used for classification by template matching after performing image registration. Other feature extraction techniques found in literature are discussed here.

In [14] the Discrete Cosine Transform and wavelets were used for feature representation. [35] GLCM (Grey Level Cooccurrence Matrix) was used for sclera biometrics. Statistical features of GLCM, such as contrast, correlation, energy and homogeneity, were used in conjunction with Fisher linear discriminant analysis. The authors in [15] presented four fusion methods for combining recognition results from multi-angle images. The combination method used here were average score, min score, max score and quality based average score.

LBP (Local Binary Pattern) feature was used for sclera biometrics in [36]. A tile-based feature extraction method was used for sclera biometrics in [37].

A feasibility study on sclera recognition was performed in [38]. Here the study was undertaken with respect to global and local information, which are extracted from the periocular region using texture and point operators resulting in a feature set that can be used for matching. Global and local information are extracted from the periocular region using texture and point operators resulting in a feature set that can be used for matching. Here SIFT descriptor was used for this purpose feature set matching. For image alignment Gradient Orientation was used. For texture based feature extraction LBP (Local Binary Patter) was employed. The effect of fusing these feature sets was also studied.

\section{Classification}

Biometric algorithms generally aim to provide a reasonable answer for all possible inputs so classification plays a important role, its importance is also reflected in sclera literature. In [10] classification was performed by template matching against the stored template for verification by one-to-one matching was used. Similarly, one-to-many matching against all the templates was used in the case of identification. Two different methods for coarse and fine level matching are used for further classification.

A feed forward Neural Network with a single hidden layer was used here for classification in [14]. In [35] match score level fusion of Fisher LDA and neural networks are used which provides the best results in classification.

For classification, template matching was used in [15]. In [2], [25] for classification, the Hamming distance was used for template-based matching. In [31] after feature extraction and matching technique SURF (Speeded Up Robust Features) was used for key point matching. Followed by a direct correlation matching by pixel to pixel matching and at last minutiae matching was done for bifurcated blood vessel matching. For fusing the feature of the different poses minutia score and direct correlation scores was used.

\section{Datasets AVAilable AND PERFormance on THEM}

Benchmark datasets are very important for any biometric recognition related research. So, datasets available and performance on them are reported incorporated in this section. Sclera vessel patters are better prominent in the images taken in visible wave lengths. The vessel patterns are not prominent in infrared images of the eye. Various in-house dataset and performance on them are listed in table I.

TABLE I. VARIOUS IN-HOUSE DATASET USED IN SCLERA BIOMETRIC RESEARCH

\begin{tabular}{cccc}
\hline $\begin{array}{c}\text { Dataset } \\
\text { in Ref. }\end{array}$ & No. of Subjects & $\begin{array}{c}\text { No. of Images/ } \\
\text { Subjects }\end{array}$ & $\begin{array}{c}\text { Accurecy(\%)/ } \\
\text { EER(\%) }\end{array}$ \\
\hline$[10]$ & 6 (Both left and right eye) & 2 & 100 \\
\hline$[14]$ & 50 (Looking left and right) & - & 4.3 \\
\hline$[40]$ & 50 (Session 1) $17($ Session 2) & $\begin{array}{c}48(\text { Session 1 } \\
\text { and Session 2 each) }\end{array}$ & 25 \\
\hline$[38]$ & 30 & $958($ all together) & 77 Rank 1 \\
\hline$[35]$ & 50 (Session 1 and 2 each) & - & 13.82 \\
\hline$[37]$ & 50 (Session 1 and 2 each) & 2 & 4.29 \\
\hline$[31]$ & 103 & 3280 & 0.37 (best reasult) \\
\hline
\end{tabular}


In [14] the EERs reported were $4.3 \%$ for near distance images ( $1 \mathrm{ft}), 8.8 \%$ for medium distance images $(5 \mathrm{ft})$, and $9.2 \%$ for long distance images $(9 \mathrm{ft})$. The performance degraded slightly when training and testing in the neural network used conjunctival vasculature images of only one eye per subject, which yielded $6.5 \%, 7.4 \%$, and $11 \%$ errors.

In [40] colour images (RGB) were collected from both eyes at three different distances: 1 foot, considered as near distance images, 9 feet as medium distance images and 12 feet as far distance images. For each eye, 2 images were collected per gaze at each distance.

In [35] more robust and efficient multi-specular sclera biometric was highlighted. A multi-specular data base was prepared for the experimentation. Images are capture in different poses like right eye looking left and right, left eye looking right and left.

A multimodal eye recognition technique in visible spectrum was explained in [37]. A weighted fusion scheme to combine the information originating from these two modalities is highlighted here. For experimentation on a dataset of different gender of varying age was used.

A sclera recognition technique for multi-spectral occluded sclera recognition was proposed in [31]. A multi-specular data base was prepared for the experimentation. Images are capture in different poses like right eye looking left and right, left eye looking right and left.

In [15], a robust multi-angled sclera recognition technique was proposed. IUPUI multi-wavelength database was acquired under eight different wavelengths illumination were used over here. For each wavelength, there are a total of 352 images of 4 different eye colours. However higher Genuine Accept Rate (GAR) can be obtain but then FAR becomes smaller, quality based-average and simple-average can be employed then. To obtain lower FAR when GAR goes to 1 and max-score fusion method was used. In [2] the IUPUI multi-wavelength dataset is also used and there a EER of 11.89 for automatic segmentation and 8.49 for manual segmentation is achieved.

It is found that only two public datasets are available for sclera biometric research, namely UBIRIS Version 1 and 2 [42]. The UBIRIS version 1 database consists of 1877 RGB images taken in two distinct sessions (1205 images in session 1 and 672 images in session 2) from 241 identities. Both high resolution images $(800 \times 600)$ and low resolution images $(200 \times 150)$ are provided in the database. In UBIRIS version-2 [42] the images were actually captured on nonconstrained conditions at-a-distance, on-the-move and on the visible wavelength. Here 261 subjects of sclera 522 are present in this version. From these subjects a total of 11,102 eye images are present in two sessions. Few subjects are there where the Volunteers are wearing glasses but this data. Various performances on UBIRIS Version 1 are listed in table II.

TABLE II. VARIOUS PERFORMANCES ON UBIRIS VERSION 1

\begin{tabular}{cc}
\hline Dataset in Ref. & $\operatorname{Accurecy}(\%) /$ EER(\%) \\
\hline$[25]$ & 3.02 \\
\hline$[39]$ & FAR $=0$ and FRR $=0.0769$ for the images of poor quality \\
\hline$[2]$ & 11.89 \\
\hline$[27]$ & 91.42 \\
\hline$[37]$ & 0.47 \\
\hline
\end{tabular}

The authors of [25] come up with the first multimodal eye recognition technique using the sclera and iris. It is also the first paper that used a public robust dataset in sclera biometric.
A kernel-based matching score fusion method was used here.

In [39], the authors propose a comprehensive sclera image quality measure, which can quickly detect if the image has a valid eye, assess the image quality, evaluate the segmentation accuracy, and measure if the image has sufficient feature information for recognition.

In order to increase the robustness of the sclera literature a quality fusion-based biometric technique using sclera and iris information was explained in [27]. Here a quality function was defined for both iris and sclera in order to combine them. Separate quality based formulation was done for entire eye image, sclera image, iris image and finally to get the overall eye image.

[36] was the first work in literature where sclera biometric cancellable identity verification was introduced. A polar coordinate mapping was used to make the images scale invariant. A mask was developed to search the overlapping region for a template match. Here a kernel is been design to implement cancellable identity verification.

\section{AFFECTS OF THE SCLERA BIOMETRIC ON MEDICAL AND OTHER CONDITIONS}

There are various medical conditions that may affect the result of sclera biometric. Because if the pattern of the sclera is been affected by any disorder or diseases. This will affect the biometric system to a large extend. Episcleritis is a common inflammatory condition seen in younger patients[41]. The overlying conjunctiva can also be swollen and red, revealing the crisscrossing pattern of the underlying congested episcleral vessels. Hence, the pattern of the sclera gets affected highly. Scleritis is a much more severe and chronic disease found in the aged person. Thus the patient presents with a red eye that can rapidly progress to a more fulminant course. Degenerations of the sclera occur commonly in response to aging and disease. As the age increase, the eyes take on a lackluster appearance due to the reduction in tear flow and a loss of episcleral tissue. This is other way by which the pattern may get affected. Other sclera disorders such as Staphyloma, Ectasia, Granulomatous Inflammations, and Pigmented Abnormalities etc. can highly affect the recognition system. Other conditions like, when a man is feeling sleepy or is drunk may also affect the pattern obtained.

\section{CONCLUSION AND FUtURE WORK}

The literature relevant to sclera biometrics is not large, but is growing rapidly and spreads across a wide variety of sources. This survey suggests a structure for the sclera biometrics literature and summarizes the current state-of-theart. There are still a number of active research topics within Sclera biometrics. Many of these are related to the desire to make sclera recognition practical in less-controlled conditions and also a real time process as much as possible. This survey also highlights the few future challenges and existing difficulties that can be addressed in further research, e.g. more research should be done to see how recognition could be improved for more robust populations and conditions. Also, research on sclera biometrics for people wearing glasses can be another challenge in this field. Another area that has not received much investigation is the combination of multiple images or the use of multiple biometrics (e.g. sclera and 
iris recognition) to improve performance. The efficiency of the matching algorithms will also become more important as sclera biometrics are deployed in recognition applications for large populations. Various medical conditions and other situations affecting the recognition discussed in this paper should be solved in future research. Further research can also be initiated for developing datasets of sclera images in multiangle images, images capturing different medical conditions and images at distance. Research work on developing synthetic sclera datasets can be another scope of investigation in this area. We hope that this survey will serve to focus more researcher attention towards the emerging sclera biometric.

\section{REFERENCES}

[1] A. K. Jain, R. Bolle, and S. Pankanti (Eds), BIOMETRICS: Personal Identification in Networked Society, Kluwer Academic Publishers, 1999.

[2] Z. Zhou, Y. Du, N. L. Thomas, and E. J. Delp, A New Human Identification Method: Sclera Recognition, IEEE transaction on System, Man And Cybernatics PART A: System And Human, vol. 42, no. 3, pp - 571-583, 2012.

[3] C. Simon, and I. Goldstein, A new scientific method of identification, New York State Journal of Medicine, vol. 35, no. 18, pp. 901-906, 1935.

[4] P. Tower, The fundus Oculi in monozygotic twins: Report of six pairs of identical twins, Archives of Ophthalmology, vol. 54, pp. 225-239, 1955.

[5] A. M. Joussen, Vascular plasticity - the role of the angiopoietins in modulating ocular angiogenesis, Graefe's Archive for Clinical and Experimental Ophthalmology, vol. 239, no 12, pp. 972 - 975, 2001.

[6] H. Kobayashi and S. Kohshima, Unique morphology of the human eye, Nature, vol. 387, no. 6635, pp. 767-768, 1997.

[7] N. Miura, A. Nagasaka, and T. Miyatake, Feature extraction of finger vein patterns based on iterative line tracking and its application to personal identification, Systems and Computers in Japan, vol. 35, pp. 61-71, 2004.

[8] C. L. Lin, and K. C. Fan, Biometric verification using Thermal images of palm-dorsa vein patterns, IEEE Transactions on Circuits and Systems for Video Technology, vol. 14, pp. 199-213, 2004.

[9] R. Hill, Retina Identification, Chapter 4 in A, Jain, Bolle, and S, Pankanti, BIOMETRICS: Personal Identification in Networked Society, 2nd Printing, Kluwer Academic Publishers, 1999.

[10] R. Derakhshani, A. Ross, and S. Crihalmeanu, A new biometric modality based on conjunctival vasculature. Proceedings of Artificial Neural Networks in Engineering, pp. 1-8, 2006.

[11] C. G. Owen, T. J. Ellis, A. R. Rudnicka, and E.G. Woodward, Optimal green (red-free) digital imaging of conjunctival vasculature. Ophthalmic Physiol Opt. vol. 22, no. 3, pp. 234-243.

[12] Q. Li, S. Sone, and K. Doi, Selective enhancement filters for nodules, vessels, and airway walls in two-and three dimentional CT scans, Medical Physics, vol. 30, no. 8, 2003.

[13] E. M. Perez, A. D. Hughes, A. V. Stanton, S. A. Tom, A. A. Bharath, and K. H. Parker, Segmentation of retinal blood vessels based on the second directional derivative and region growing, International Conference on Image Processing (ICIP), vol. 2, pp. 173-176, 1999.

[14] R. Derakhshani and A. Ross, A texture-based neural network classifier for biometric identification using ocular surface vasculature, Proc. of International Joint Conference on Neural Networks, pp. 2982-2987, 2007.

[15] Z. Zhou, Y. Du, N. L. Thomas, and E. J. Delp, Multi-angle Sclera Recognition System, IEEE Workshop on Computational Intelligence in Biometrics and Identity Management: pp. 103 -108, 2011.

[16] J. Daugman, How iris recognition works, IEEE Transactions onCircuits and Systems for Video Technology, vol. 14, no. 1, pp. 21-30, 2004

[17] J. Daugman, New Methods in Iris Recognition, Systems, Man, and Cybernetics, Part B, IEEE Transactions on, vol. 37, no. 5, pp. 11671175, 2007.

[18] W. W. Boles and B. Boashash, A human identification technique using images of the iris and wavelet transform, Signal Processing, IEEE Transactions on, vol. 46, no. 4, pp. 1185-1188, 1998.
[19] X. Liu, K.W. Bowyer and P. J. Flynn, Experiments with an improved iris segmentation algorithm, Proc. Automatic Identification Advanced Technologies,Fourth IEEE Workshop on, pp. 118-123, 2005.

[20] R.P. Wildes, Iris recognition: an emerging biometric technology, Proc. of the IEEE, vol. 85, no. 9, pp. 1348-1363, 1997.

[21] G.O. Williams, Iris recognition technology, Aerospace and Electronic Systems Magazine, IEEE, vol. 12, no. 4, pp. 23-29, 1997.

[22] M. Li, T. Tieniu, W. Yunhong and Z. Dexin, Efficient iris recognition by characterizing key local variations, IEEE Transactions on Image Processing, vol. 13, no. 6, pp. 739-750, 2004.

[23] Q.-C. Tian, Q. Pan, Y.-M. Cheng and Q.-X. Gao, Fast algorithm and application of Hough transform in iris segmentation, Proc. Machine Learning and Cybernetics, vol. 3977, pp. 3977-3980, 2004.

[24] N. V. Huan and H. Kim, A Novel Circle Detection Method for Iris Segmentation, Proc. Image and Signal Processing, pp. 620-624, 2008.

[25] Z. Zhou, Y. Du, N. L. Thomas, and E. J. Delp, Multimodal eye recognition. Proc. of the International Society for Optical Engineering, vol.7708, no. 770806, pp. 1-10, 2010.

[26] S. Crihalmeanu, A. Ross and R. Derakhshani, Enhancement and Registration Schemes for Matching Conjunctival Vasculature, Series Enhancement, Springer-Verlag, LNCS 5558, pp. 1247-1256, 2009.

[27] Z. Zhou, Y. Du, N. L. Thomas, and E. J. Delp, Quality Fusion Based Multimodal Eye Recognition, IEEE International Conference on Systems, Man, and Cybernetics: pp. 1297-1302, 2012.

[28] M. H. Khosravi and R. Safabakhsh, Human eye sclera detection and tracking using a modified time-adaptive self-organizing map, Pattern Recognition, vol. 41, pp 2571 -2593, 2008.

[29] H. Shah-Hosseini, R. Safabakhsh, TASOM: a new time adaptive self organizing map, IEEE Trans. Syst. Man Cybern. Part B, vol. 33 ,no. 2, pp. 271-282, 2003.

[30] H. Shah-Hosseini, R. Safabakhsh, A TASOM-based algorithm for active contour modelling, Pattern Recognition Letter, vol. 24 , no. 9, pp. 136113732003.

[31] S. Crihalmeanu and A. Ross, Multispectral sclera patterns for ocular biometric recognition, Pattern Recognition Letters, vol 33, pp. 18601869, 2012.

[32] C. J. Tucker, Red and photographic infrared linear combinations for monitoring vegetation. Remote Sens. Environment. vol. 8, pp. 127-150, 1979.

[33] S. Crihalmeanu, A. Ross,. On the use of multispectral conjunctival vasculature as a soft biometric. In: Proc. IEEE Workshop on Applications of Computer Vision (WACV), pp. 204-211, 2011.

[34] L. Qiang, , S. Shusuke, D. Kunio, Selective enhancement filters for nodules, vessels, and airway walls in two or three dimentional CT scans. Medical. Physics. vol.30, no. 8, pp. 2040-2051, 2003.

[35] S. P. Tankasala, P. Doynov, R. R. Derakhshani, A. Ross and S. Crihalmeanu, Biometric Recognition of Conjunctival Vasculature using GLCM Features, International Conference on Image Information Processing, pp. 1-6, 2011.

[36] K. Oh and K. Toh, Extracting Sclera Features for Cancelable Identity Verification, 5th IAPR International Conference on Biometric, pp. 245250,2012

[37] V. Gottemukkula, S. K. Saripalle, S. P. Tankasala, R. Derakhshani, R. Pasula and A. Ross, Fusing Iris and Conjunctival Vasculature: Ocular Biometrics in the Visible Spectrum, IEEE Conference on Technologies for Homeland Security, pp. 150-155, 2012.

[38] U. Park, A. Ross, and A. K. Jain, Periocular Biometrics in the Visible Spectrum: A Feasibility Study, IEEE 3rd International Conference on Biometrics: Theory, Applications, and Systems , pp. 1-6, 2009.

[39] Z. Zhou, E. Y. Du, and N. L. Thomas, A Comprehensive Sclera Image Quality Measure, 11th International Conference on Control, Automation, Robotics and Vision, pp. 638-643, 2010.

[40] S. Crihalmeanu , A. Ross, and R. Derakhshani, Enhancement and Registration Schemes for Matching Conjunctival Vasculature, Appeared in Procceding of the 3rd IAPR/IEEE International Conference on Biometrics, pp.1247-1256, 2009

[41] R. M. Kershner, Disorders of Sclera, Available at. http://www.asiteforeyes.com/DisordersoftheSclera.htm

[42] UBIRIS version 1 and 2 dataset, Available at ://iris.di.ubi.pt/ubiris.html 